\title{
Speech Perception and Information-Carrying Capacity for Hearing Aid Users of Different Ages
}

\author{
Ulrich Hoppe ${ }^{a}$ Thomas Hocke $^{b}$ Alexander Müller ${ }^{c}$ Anne Hast ${ }^{a}$ \\ ${ }^{a}$ Department of Audiology, ENT Clinic, University of Erlangen-Nürnberg, Erlangen, ${ }^{\mathrm{b}}$ Cochlear Deutschland \\ GmbH \& Co., Hannover, ' Vivantes Hearing Center, ORL Department Friedrichshain Clinic, Berlin, Germany
}

\section{Key Words}

Aging $\cdot$ Speech perception $\cdot$ Hearing aid use $\cdot$ Monosyllabic score

\begin{abstract}
Hearing impairment in the elderly is usually treated with conventional hearing aids; however, a large number of older people do not achieve sufficient speech recognition with hearing aids. The aim of the study was to describe speech perception with hearing aids in comparison to pure-tone hearing loss and maximum speech recognition scores for phonemically balanced words. Data from 392 hearing aid users with different degrees of hearing loss were evaluated retrospectively. In particular, pure-tone thresholds, the maximum monosyllabic word score, and the monosyllabic word score in quiet at conversational level with a hearing aid were analysed. The results showed that speech perception scores decline with increasing age. Even when corrected for puretone hearing loss, a significant decline in speech recognition scores after the age of 80 years was observed. Regarding the maximum monosyllabic word score, the effect is smaller but still observable; thus, speech recognition with hearing aids is significantly lower for older subjects. This can be attributed partially to the reduction of the information-carrying capacity in this group.

(C) 2016 The Author(s)

Published by S. Karger AG, Basel
\end{abstract}

\section{Introduction}

Deterioration in hearing ability that occurs with aging is a complex process that can vary in severity from minor to substantial. According to van Rooij et al. [1990], the proportion of elderly persons with problems in perceiving speech doubles per decade of life from $16 \%$ at the age of 60 to $32 \%$ at the age of 70 and to $64 \%$ at the age of 80 . According to the ISO report for thresholds of hearing loss versus age, every second person above 65 years of age needs hearing aid (HA) amplification. Left untreated, presbyacusis of a moderate or greater degree affects communication and reduces quality of life substantially. Where HAs no longer provide benefit, cochlear implantation is the treatment of choice with excellent results even in elderly subjects [Hast et al., 2015].

Most severely hearing-impaired adults have a history of some HA use where they have experienced steadily diminishing speech perception ability. As the main goal of cochlear implantation is the improvement of speech understanding, beyond what is feasible by conventional amplification, a thorough HA evaluation is advisable prior to cochlear implant (CI) surgery. One important outcome measure in HA evaluations is the perception of monosyllabic words at a conversational level. An individual's speech recognition score with HAs at conversational level $\left[\mathrm{SRS}_{65}(\mathrm{HA})\right]$ varies dramatically with pure-tone hearing

\section{KARGER \\ E-Mail karger@karger.com www.karger.com/aud}

\section{(9) 2016 The Author(s) \\ Published by S. Karger AG, Basel $1420-3030 / 16 / 0217-0016 \$ 39.50 / 0$}

This article is licensed under the Creative Commons AttributionNonCommercial-NoDerivatives 4.0 International License (CC BYNC-ND) (http://www.karger.com/Services/OpenAccessLicense) Usage and distribution for commercial purposes as well as any distribution of modified material requires written permission.
Prof. Dr. Dr. Ulrich Hoppe

Department of Audiology

ENT Clinic, University of Erlangen-Nürnberg

Waldstrasse 1, DE-91054 Erlangen (Germany)

E-Mail ulrich.hoppe@uk-erlangen.de 


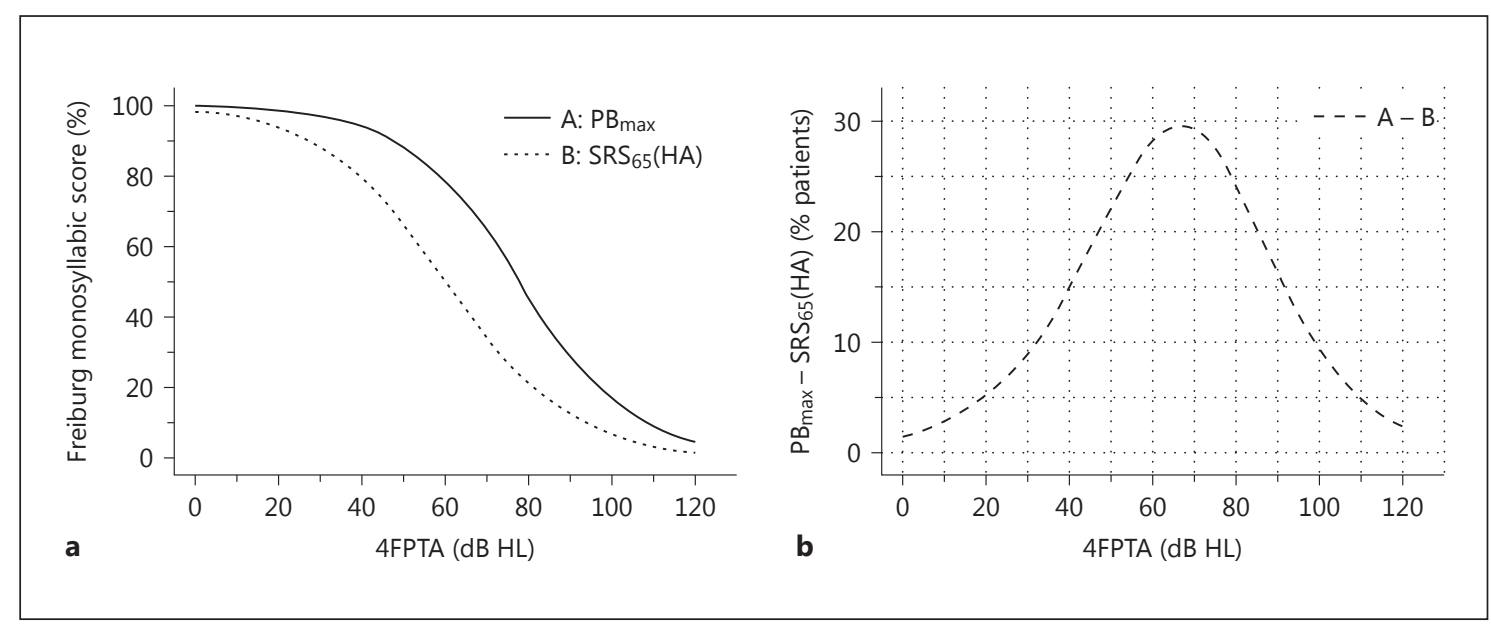

Fig. 1. a Speech recognition score for Freiburg monosyllabic words as a function of pure-tone loss measured as pure-tone average for thresholds at $0.5,1,2$, and $4 \mathrm{kHz}(4 \mathrm{FPTA})$. The solid line represents $\mathrm{PB}_{\max }$, and the dotted line represents scores at $65 \mathrm{~dB}$ with $\mathrm{HAs}\left[\mathrm{SRS}_{65}(\mathrm{HA})\right]$. b Difference of the functions shown above $\left[\mathrm{PB}_{\max }-\right.$ $\left.\mathrm{SRS}_{65}(\mathrm{HA})\right]$.

loss. However, the average dependency between the two variables can be characterized by a logistic function [Hoppe et al., 2014, 2015] with an anticipated 100\% at mild hearing losses and nearly zero for profound hearing loss. This function is shown in figure $1 \mathrm{a}$. In a prior study [Hoppe et al., 2014], a similar function was found for the maximum phonemically balanced word score $\left(\mathrm{PB}_{\max }\right)$ measured under headphones (fig. 1a, dotted line).

The $\mathrm{PB}_{\max }$ defines the maximum monosyllabic word recognition score for phonemically balanced words [Gelfand, 1997]. According to Halpin and Rauch [2008], the $\mathrm{PB}_{\max }$ can be used as an estimator for the informationcarrying capacity (ICC) of the auditory system. Within the process of $\mathrm{HA}$ evaluation, the $\mathrm{PB}_{\max }$ characterizes the potential for the best possible speech recognition with HAs at a conversational level of $65 \mathrm{~dB}$ sound pressure level (SPL). As shown in figure 1, not all HA users can transfer their full ICC into speech recognition scores using conventional amplification, and a gap of up to $30 \%$ remains. Figure $1 b$ shows that this gap depends on the amount of pure-tone hearing loss with a maximum reached for severe hearing loss levels. For severe hearing losses, the potential HA performance is limited by an insufficient residual dynamic range [Zwartenkot et al., 2014] and the aversiveness to the high levels of acoustic amplification [Hoppe et al., 2014]. Additionally, the effect of aging on cognitive processes is a major contributing factor affecting HA performance [Walden and Walden, 2004; Müller et al., 2016].

Speech Perception in Elderly Hearing Aid Users
The aim of our retrospective analysis was a detailed investigation of the age dependency of the ICC. Another question was whether the perceptual gap between $\mathrm{PB}_{\max }$ and $\mathrm{SRS}_{65}(\mathrm{HA})$ depends on the subject's age.

\section{Methods}

In total, $\mathrm{HA}$ evaluations of 392 subjects with bilateral sensorineural hearing loss performed in the Audiological Centres of Erlangen $(\mathrm{n}=156)$ and Berlin $(\mathrm{n}=236)$ were analysed retrospectively. The data from 784 ears were analysed separately. The mean age of the subjects was $65 \pm 16$ years and ranged from 18 to 96 years.

The data were collected as part of clinical routine for HA performance assessment. All subjects had been supplied with HAs and had at least 3 months of experience using them. Approximately $50 \%$ of the subjects were first-time users. All had completed the HA selection and fitting process at least 3 months before the visit. Prior to aided hearing assessment, the HAs were technically checked. All patients attended a follow-up visit with their HA professional before the evaluation visit. Only native German-speaking subjects with no known mental disabilities influencing audiometric test results were included in the analyses. Subjects with air-bone gaps of more than $5 \mathrm{~dB}$ were excluded from the analyses.

Each ear was tested separately. The pure-tone average threshold for the frequencies $0.5,1,2$ and $4 \mathrm{kHz}$ was calculated for each ear. Speech audiometry using Freiburg monosyllabic words was measured via headphones in the unaided condition for each ear. The speech stimuli were presented at $65 \mathrm{~dB}$ SPL initially, and the corresponding speech recognition score, i.e. correct word score in percent, was recorded. The presentation level was increased stepwise by $10-15 \mathrm{~dB}$ until a maximum score of $100 \%$ was achieved or

Audiol Neurotol 2016;21(suppl 1):16-20 
Fig. 2. Scatter representation of the maximum monosyllabic score $\left(\mathrm{PB}_{\max }\right)$ as a function of 4 -frequency average at $0.5,1,2$, and $4 \mathrm{kHz}$ (4FPTA). Each dot represents the result from one ear.

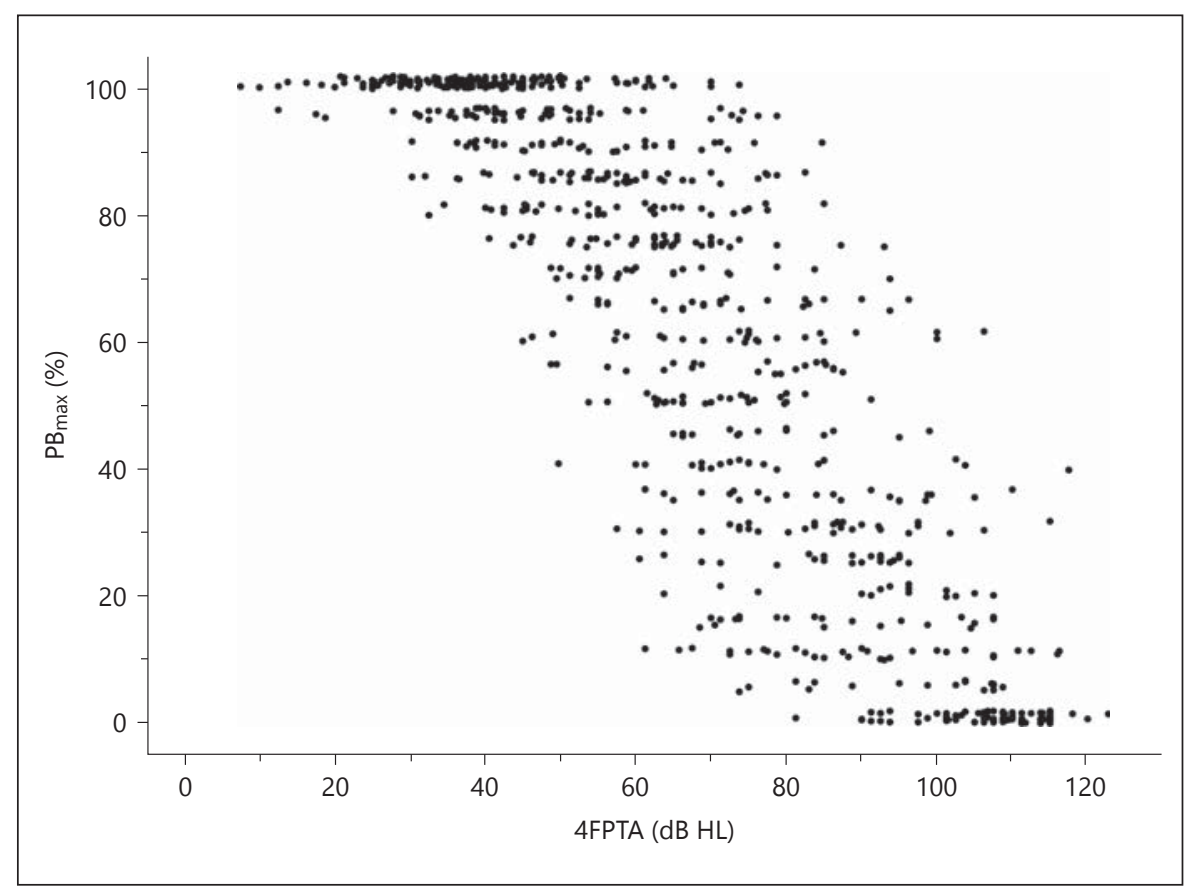

With increasing age for each subgroup, a tendency for larger discrepancies from the average performance is observed. For those below 70 years of age, no systematic deviation from the average performance line can be found. Above 70 years of age, a clear trend for decreasing performance is present. Discrepancies to the average performance increase from $-3 \%$ (70-80 years) to $-7 \%$ ( $80-90$ years) and $-18 \%$ ( $>90$ years). Additionally, the variability within each age group increases with increasing age: interquartile ranges vary from $15-17 \%$ for younger groups to $26 \%$ for the group aged $80-90$ years.

\section{Discussion}

Successful decoding of speech signals requires functional integrity at all stages along the auditory pathway including the inner ear, cochlear nerve, brainstem, and ultimately the auditory cortex. From the functional point of view, not only is sensitivity important, but also sufficient temporal and spectral resolutions are required for speech recognition and comprehension.

This study investigated the deterioration of speech recognition in the elderly. While there is a great deal of knowledge about the loss of threshold sensitivity for pure tones with increasing age, little is known about the progressive decline of speech recognition in the elderly. The study shows that speech recognition is affected to a great-
18 DOI: $10.1159 / 000448349$
Hoppe/Hocke/Müller/Hast 
Fig. 3. Difference between individual $\mathrm{PB}_{\max }$ scores and average performance $(\mathrm{A})$ as a function of age. Box plots summarize data of one decade from 40 to 90 years. Figures within the box plots represent the number of ears within the range.

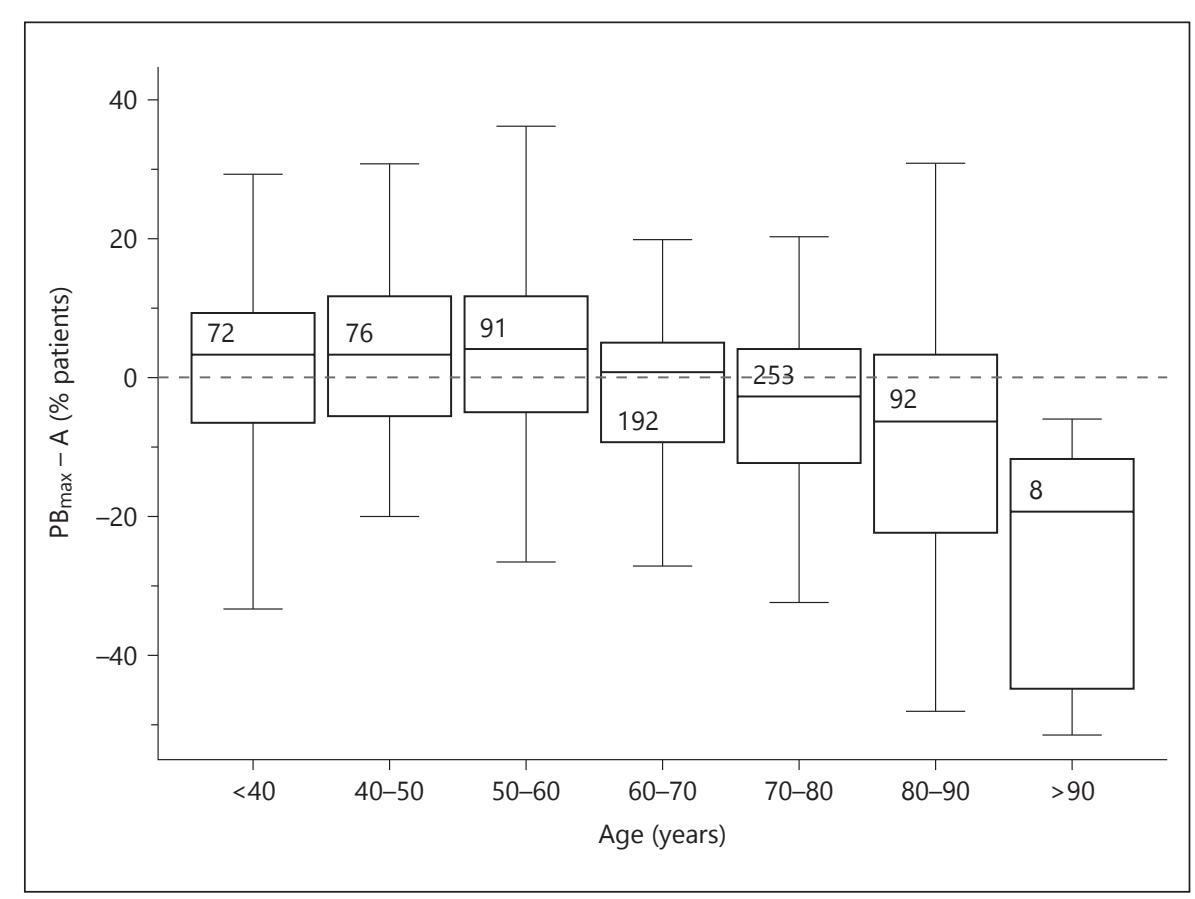

er extent than would be predicted from the pure-tone thresholds, alone, for the older elderly group of $>80$ years of age. In particular, the $\mathrm{PB}_{\max }$, a measure of the ICC, is significantly reduced in this group compared to the younger elderly group.

Recent reports have demonstrated that aided speech perception for older HA users is significantly lower than for younger adults [Hoppe et al., 2014; Müller et al., 2016]. Though HA fittings were technically verified, older HA listeners $\geq 70$ years achieved speech recognition scores that were $10-20 \%$ lower than for younger HA listeners. The primary hypothesis was that the HA users in the more senior groups do not receive the appropriate amplification and sound quality from their HAs. Our study, however, shows that even when presenting amplified speech stimuli via headphones, the speech recognition score is significantly reduced in the older elderly group compared to the younger elderly groups. This reduced ICC for older listeners is supported by models of degeneration of the central auditory pathway that indicate that feature extraction and pattern recognition for auditory patterns such as speech are reduced in the elderly [Humes, 1996]. Currently, the question remains open whether the reduced speech recognition depends on cognitive factors located in the cortex or on auditory signal processing dysfunction taking place at cochlear or brainstem levels.

Speech Perception in Elderly Hearing Aid Users
On the other hand, a number of studies indicate that older subjects who are provided with a CI can achieve speech perception scores that are identical or very similar to younger CI users [Nakajima et al., 2000; Orabi et al., 2006; Clark et al., 2012; Hast et al., 2015]. Further, elderly CI listeners also demonstrate benefit from individually tailored auditory training therapy that can result in auditory outcomes marginally below those observed for younger CI users [Schumann et al., 2014].

The use of the $\mathrm{PB}_{\max }$ as an estimator of the ICC was first proposed by Halpin and Rauch [2008]. They demonstrated that the $\mathrm{PB}_{\max }$ is a more valid measure for an estimate of everyday speech recognition than the pure-tone threshold levels in subjects with sensorineural hearing loss. As shown in figure 2, identical averaged pure-tone hearing losses result in a very different ICC. Obviously, the 4 -frequency pure-tone average is a rough measure only for overall hearing ability and considers neither influencing temporal aspects nor the frequency characteristics of a hearing loss; therefore, the $\mathrm{PB}_{\max }$ provides important additional information. However, when estimating speech perception in everyday life one has to consider that the $\mathrm{PB}_{\max }$ is not achieved in many cases. As shown in figure 1, differences between $\mathrm{PB}_{\max }$ and speech recognition scores with HA at $65 \mathrm{~dB}$ SPL can be as large as $30 \%$. Considering the simple measurement of the $\mathrm{PB}_{\max }$, it provides an excellent tool when screening for CI candidacy 
[Hoppe et al., 2015]. As an example, when a subject's $\mathrm{PB}_{\max }$ is below $60 \%$ for amplified speech under headphones for monosyllabic words, it is feasible that their corresponding monosyllabic word score at conversational level via an HA will be $30 \%$ or lower. Such poor performance with conventional amplification would clearly make this individual a candidate for $\mathrm{CI}$ according to the commonly applied audiological criterion across CI clinics. Clinically, evaluation of the $\mathrm{PB}_{\max }$ requires measurements by the standard audiometer and headphones only and is independent of the actual HA settings. As such the $\mathrm{PB}_{\max }$ provides a simple and readily available method to all audiology centres for the identification of potential CI candidates amongst the hearing-impaired elderly population for further referral to and evaluation by specialist implant teams.

\section{Conclusion}

The $\mathrm{PB}_{\max }$ provides a valid estimator for the ICC and hence also the maximum ability for speech recognition and understanding in everyday life. In contrast to puretone thresholds, it considers both temporal aspects and suprathreshold auditory processing involved in speech perception. Therefore, estimation of the ICC via measurement of the $\mathrm{PB}_{\max }$ should be used routinely during the evaluation for CI candidacy for patients of any age.

\section{Disclosure Statement}

T. Hocke is an employee of Cochlear Deutschland $\mathrm{GmbH} \&$ Co. KG. The other authors (U.H., A.M., A.H.) state that there is no conflict of interest to be disclosed.

\section{References}

Clark JH, Yeagle J, Arbaje AI, et al: Cochlear implant rehabilitation in older adults: Literature review and proposal of a conceptual framework. J Am Geriatr Soc 2012;60:1936-1945.

Gelfand SA: Essential of Audiology. New York, Thieme, 1997.

Halpin C, Rauch SD: Clinical implications of a damaged cochlea: pure tone thresholds vs information carrying capacity. Otolaryngol Head Neck Surg 2008;140:473-476.

Hast A, Schlücker L, Digeser F, Liebscher T, Hoppe U: Speech perception of elderly cochlear implant users under different noise conditions. Otol Neurotol 2015;36:16381643.

Hoppe U, Hast A, Hocke T: Speech perception with hearing aids in comparison to pure-tone hearing loss. HNO 2014;62:443-448.
Hoppe U, Hast A, Hocke T: Audiometry-based screening procedure for cochlear implant candidacy. Otol Neurotol 2015;36:10011005.

Humes L: Speech understanding in the elderly. J Am Acad Audiol 1996;7:161-167.

Müller A, Hocke T, Hoppe U, Mir-Salim P: The age effect in evaluation of hearing aid benefits by speech audiometry. HNO 2016;64:143148.

Nakajima S, Iwaki S, Fujisawa N, Yamaguchi S, Kawano M, Fujiki N, Naito Y, Honjo I: Speech discrimination in elderly cochlear implant users. Adv Otorhinolaryngol 2000;57:393-396.

Orabi AA, Mawman D, Al-Zoubi F, Saeed SR, Ramsden RT: Cochlear implant outcomes and quality of life in the elderly: Manchester experience over 13 years. Clin Otolaryngol 2006;31:116-122.
Schumann A, Hast A, Hoppe U: Speech performance and training effects in the cochlear implant elderly. Audiol Neurotol 2014;19:4548.

Van Rooij J, Plomp R, Orlebeke J: Auditive and cognitive factors in speech perception by elderly listeners. II. Multivariate analysis. J Acoust Soc Am 1990;88:2611-2624.

Walden TC, Walden BE: Predicting success with hearing aids in everyday living. J Am Acad Audiol 2004; 15:342-352.

Zwartenkot JW, Snik AF, Mylanus EA, Mulder JJ: Amplification options for patients with mixed hearing loss. Otol Neurotol 2014;35:221-226. 